\title{
New CAST limit on the axion-photon interaction
}

\author{
CAST Collaboration ${ }^{\dagger}$
}

\begin{abstract}
Hypothetical low-mass particles, such as axions, provide a compelling explanation for the dark matter in the universe. Such particles are expected to emerge abundantly from the hot interior of stars. To test this prediction, the CERN Axion Solar Telescope (CAST) uses a $9 \mathrm{~T}$ refurbished Large Hadron Collider test magnet directed towards the Sun. In the strong magnetic field, solar axions can be converted to X-ray photons which can be recorded by X-ray detectors. In the 2013-2015 run, thanks to low-background detectors and a new X-ray telescope, the signal-to-noise ratio was increased by about a factor of three. Here, we report the best limit on the axion-photon coupling strength $\left(0.66 \times 10^{-10} \mathrm{GeV}^{-1}\right.$ at $95 \%$ confidence level) set by CAST, which now reaches similar levels to the most restrictive astrophysical bounds.
\end{abstract}

A dvancing the low-energy frontier is a key endeavour in the worldwide quest for particle physics beyond the standard model and in the effort to identify dark matter ${ }^{1,2}$. Nearly massless pseudoscalar bosons, often generically called axions, are particularly promising because they appear in many extensions of the standard model. They can be dark matter in the form of classical field oscillations that were excited in the early universe, notably by the re-alignment mechanism ${ }^{3}$. One particularly well motivated case is the quantum chromodynamics (QCD) axion, the eponym for all such particles. The existence of this new lowmass boson follows from the Peccei-Quinn mechanism as an explanation why QCD is perfectly time-reversal invariant within current experimental precision ${ }^{3}$.

Axions were often termed 'invisible' because of their extremely feeble interactions, yet they are the target of a fast-growing international landscape of experiments. Numerous existing and foreseen projects assume that axions are the galactic dark matter and use a variety of techniques that are sensitive to different interaction channels and optimal in different mass ranges ${ }^{4}$. Independently of the dark-matter assumption, one can search for new forces mediated by these low-mass bosons ${ }^{5}$ or the back-reaction on spinning black holes (superradiance) ${ }^{6}$. Stellar energy-loss arguments provide restrictive limits that can guide experimental efforts, and in some cases may even suggest new loss channels ${ }^{3,7,8}$.

The least model-dependent search strategies use the production and detection of axions and similar particles by their generic twophoton coupling. It is given by the vertex $\mathcal{L}_{a \gamma}=-(1 / 4) g_{a \gamma} F^{\mu \nu} \widetilde{F}_{\mu \nu} a=$ $g_{a \gamma} \mathbf{E} \cdot \mathbf{B} a$, where $a$ is the axion field, $F$ the electromagnetic field-strength tensor, and $g_{a \gamma}$ a coupling constant of dimension $(\text { energy })^{-1}$. Notice that we use natural units with $\hbar=c=k_{\mathrm{B}}=1$. This vertex enables the decay $a \rightarrow \gamma \gamma$, the Primakoff production in stars-that is, the $\gamma \rightarrow a$ scattering in the Coulomb fields of charged particles in the stellar plasma-and the coherent conversion $a \leftrightarrow \gamma$ in laboratory or astrophysical $B$-fields $s^{9,10}$.

The helioscope concept, in particular, uses a dipole magnet directed at the Sun to convert axions to X-rays (see Fig. 1 for a sketch). Solar axions emerge from many thermal processes, depending on their model-dependent interaction channels. We specifically consider axion production by Primakoff scattering of thermal photons deep in the Sun, a process that depends on the same coupling constant, $g_{a \gamma}$, which is also used for detection.

Since 2003, the CERN Axion Solar Telescope (CAST) has explored the $m_{a}-g_{a \gamma}$ parameter space with this approach (more details to be given below). The black solid line in Fig. 2 is the envelope of all previous CAST results. The low-mass part $m_{a} \lesssim 0.02 \mathrm{eV}$ corresponds to the first phase 2003-2004 using evacuated magnet bores ${ }^{11,12}$. The $a \rightarrow \gamma$ conversion probability in a homogeneous $B$ field over a distance $L$ is

$$
P_{a \rightarrow \gamma}=\left(g_{a \gamma} B \frac{\sin (q L / 2)}{q}\right)^{2}
$$

where $q=m_{a}^{2} / 2 E$ is the $a-\gamma$ momentum transfer in vacuum. For $L=9.26 \mathrm{~m}$ and energies of a few $\mathrm{keV}$, coherence is lost for $m_{a} \gtrsim 0.02 \mathrm{eV}$, explaining the loss of sensitivity for larger $m_{a}$.

Later, CAST has explored this higher-mass range by filling the conversion pipes with ${ }^{4} \mathrm{He}$ (refs 13,14 ) and ${ }^{3} \mathrm{He}$ (refs 15,16$)$ at variable pressure settings to provide photons with a refractive mass, and in this way match the $a$ and $\gamma$ momenta. The sensitivity is smaller because, at each pressure setting, data were typically taken for a few hours only. Despite this limitation, CAST has reached realistic QCD axion models and has superseded previous solar axion searches using the helioscope $e^{17}$ and Bragg scattering technique ${ }^{18,19}$. (For a more complete list of previous solar axion constraints see ref. 1.) The CAST data were also interpreted in terms of other assumed axion production channels in the Sun ${ }^{20-22}$. Moreover, CAST constraints on other low-mass bosons include chameleons ${ }^{23}$ and hidden photons ${ }^{24}$.

During this long experimental programme, CAST has used a variety of detection systems at both magnet ends, including a multiwire time projection chamber ${ }^{25}$, several Micromegas detectors ${ }^{26}$, a low-noise charged coupled device attached to a spare X-ray telescope (XRT) from the ABRIXAS X-ray mission ${ }^{27}$, a $\gamma$-ray calorimeter $^{21}$, and a silicon drift detector ${ }^{23}$.

In the latest data-taking campaign (2013-2015), CAST has returned to evacuated pipes, with an improvement in the sensitivity to solar axions of about a factor of three in signal-tonoise ratio over a decade ago, thanks to the development of novel detection systems-notably new Micromegas detectors with lower background levels, as well as a new XRT built specifically for axion searches. These developments are also part of the activities to define the detection technologies suitable for the proposed much larger next-generation axion helioscope $\mathrm{IAXO}^{28}$. We here report the results of this effort in CAST.

\section{Experiment and data taking}

CAST has utilized an LHC prototype dipole magnet ${ }^{29}$ (magnetic field $B \sim 9 \mathrm{~T}$, length $L=9.26 \mathrm{~m}$ ) with two parallel straight pipes (cross-sectional area $S=2 \times 14.5 \mathrm{~cm}^{2}$ ). The magnet is mounted on 


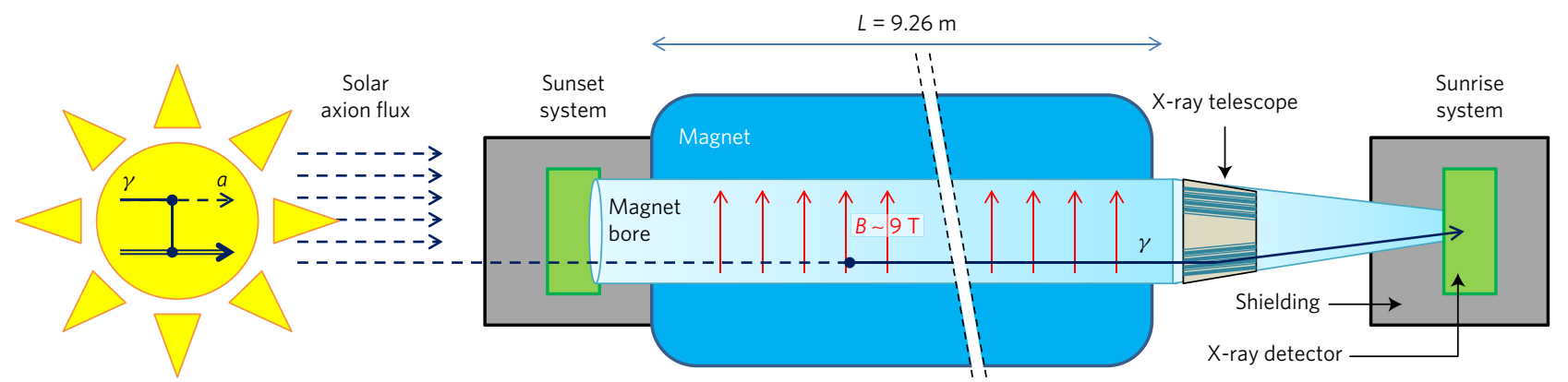

Figure 1 | Sketch of the CAST helioscope at CERN to search for solar axions. These hypothetical low-mass bosons are produced in the Sun by Primakoff scattering on charged particles and converted back to X-rays by the same process in the B-field of an LHC test magnet. The two straight conversion pipes have a cross-section of $14.5 \mathrm{~cm}^{2}$ each. The magnet can move by $\pm 8^{\circ}$ vertically and $\pm 40^{\circ}$ horizontally, enough to follow the Sun for about $1.5 \mathrm{~h}$ at dawn and dusk with each end of the magnet, where separate detection systems can search for axions at sunrise and sunset, respectively. The sunrise system is equipped with an X-ray telescope (XRT) to focus the signal on a small detector area, strongly increasing signal to noise. Our new results were achieved thanks to an XRT specifically built for CAST and improved low-noise X-ray detectors.

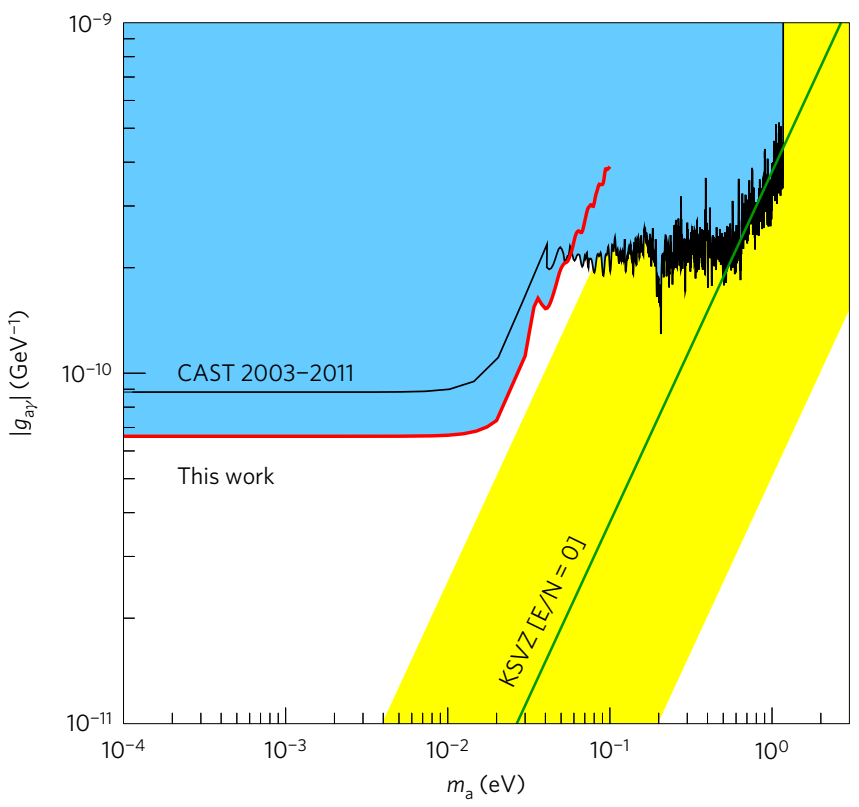

Figure 2 | CAST excluded region $(95 \% \mathrm{CL})$ in the $m_{a}-g_{a \gamma}-$ plane. Solid black line: Envelope of all CAST results from 2003-2011 data ${ }^{11-16}$. Solid red line: Exclusion from the data here presented. Diagonal yellow band: Typical QCD axion models (upper and lower bounds set according to a prescription given in ref. 49). Diagonal green line: The benchmark KSVZ axion model with $E / N=0$, where $g_{a \gamma}=(E / N-1.92) \alpha /\left(2 \pi f_{a}\right)$, with $f_{a}$ the axion decay constant.

a movable platform ( $\pm 8^{\circ}$ vertical and $\pm 40^{\circ}$ horizontal movement), allowing it to follow the Sun for about $1.5 \mathrm{~h}$ both at sunrise and sunset during the whole year. The pointing accuracy of the system is monitored to be well below $10 \%$ of the solar radius, both by periodic geometric surveys, as well as, twice per year, by filming the Sun with an optical telescope and camera attached to the magnet, and whose optical axis has been set parallel to it. The effect of refraction in the atmosphere, relevant for photons, but not for axions, is properly taken into account. At both ends of the magnet, different $\mathrm{X}$-ray detectors have been searching for photons coming from axion conversion inside the magnet when it is pointing to the Sun. During non-tracking time, calibrations are performed and detectors record background data.

The data presented here were taken with three detection systems. On the sunset (SS) side of the magnet, two gas-based lowbackground detectors (SS1 and SS2) read by Micromegas planes ${ }^{30}$, were directly connected to each of the magnet pipes. On the sunrise (SR) side of the magnet, an improved Micromegas detector was situated at the focal plane of the new XRT. The detectors were small gaseous time projection chambers of $3 \mathrm{~cm}$ drift and were filled with a 1.4 bar argon-2\% isobutane mixture. Their cathodes were $4-\mu \mathrm{m}-$ thick mylar windows that face the magnet pipe vacuum and hold the pressure difference while being transparent to X-rays. The detector parts were built with carefully selected low-radioactivity materials, and surrounded by passive (copper and lead) and active $(5-\mathrm{cm}$ thick plastic scintillators) shielding. The Micromegas readouts were built with the microbulk technique ${ }^{31}$, out of copper and kapton, and were patterned with $500 \mu \mathrm{m}$ pixels interconnected in the $x$ and $y$ directions ${ }^{32}$. These design choices are the outcome of a longstanding effort to understand and reduce background sources in these detectors ${ }^{33,34}$. This effort has led to the best background levels $\left(\sim 10^{-6} \mathrm{keV}^{-1} \mathrm{~cm}^{-2} \mathrm{~s}^{-1}\right)$ ever obtained in CAST.

The XRT installed in the SR system was a telescope (of focal length $1.5 \mathrm{~m}$ ) that follows a cone-approximation Wolter I design. It is comprised of thermally formed glass substrates deposited with $\mathrm{Pt} / \mathrm{C}$ multilayers to enhance X-ray reflectivity in the $0.5-10 \mathrm{keV}$ band. The techniques and infrastructure used in fabricating the CAST XRT were originally developed ${ }^{35}$ to make the two hard X-ray telescopes that are flying on NASA's NuSTAR satellite ${ }^{36}$. The optical prescription and multilayer coatings were optimized when considering factors including: the physical constraints of the CAST experiment; the predicted axion spectrum; and the quantum efficiency of the Micromegas detector ${ }^{37}$. The point spread function (PSF) and effective area (that is, throughput) of the XRT were calibrated at the PANTER X-ray test facility at MPE in Munich in July 2016. These calibration data were incorporated into Monte Carlo geometric ray-trace simulations to determine the expected two-dimensional (2D) distribution of solar axion-induced photons, which is shown in Fig. 3. Although there is a slight energy dependence on the PSF (the XRT focuses better at higher X-ray energy), more than $50 \%$ of the flux is always concentrated in an area of a few $\mathrm{mm}^{2}$, effectively reducing the background to levels down to $\sim 0.003$ counts $\mathrm{h}^{-1}$. In addition, the combined XRT and detector system was regularly calibrated in CAST using an X-ray source placed $\sim 12 \mathrm{~m}$ away from the optics (at the SS side of the magnet). One such calibration is shown in Fig. 3, together with the expected 2D distribution from the ray-trace simulation. These contours are different from the ones expected from axion-induced photons (shown in Fig. 3) due to the different angular size and distance of the source. The agreement between data and simulations confirms our good understanding of the optics performance. This is the first time an XRT has been designed and built specifically for axion physics and operated together with a Micromegas detector at 

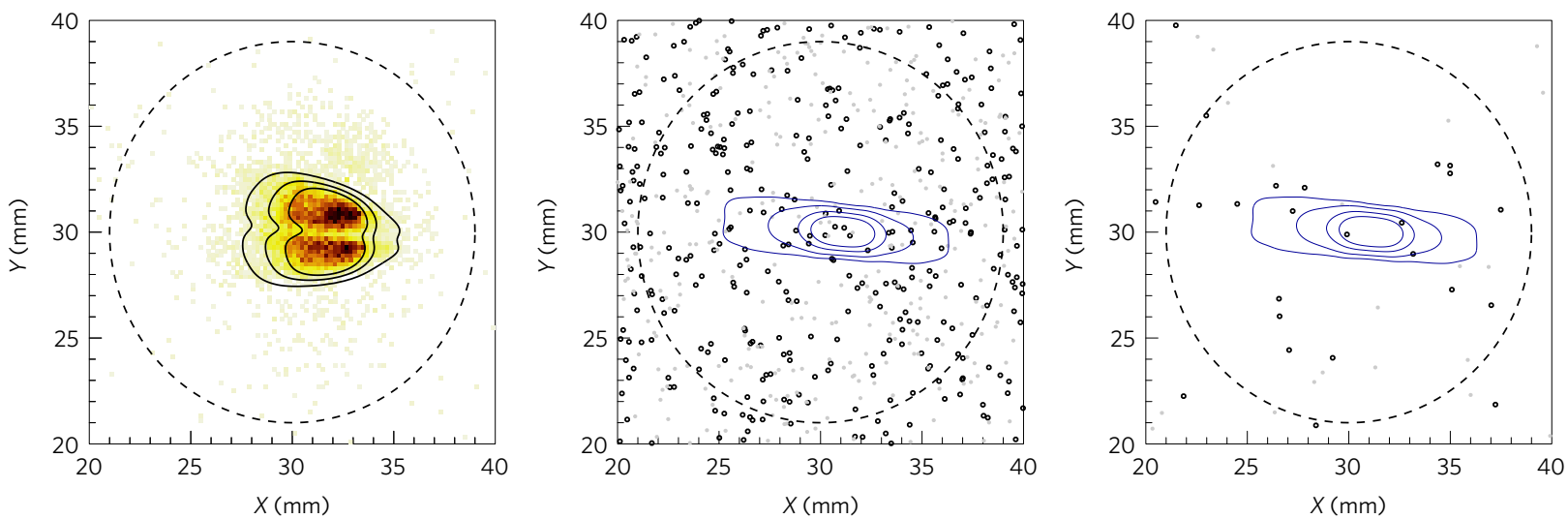

Figure 3 | 2D hitmap of events detected in the sunrise detector in a typical in situ calibration run (left), as well as in the background (middle) and tracking (right) data (both $\mathrm{K}$ and $\mathrm{L}$ data sets of Table 1). The calibration is performed with an X-ray source placed $\sim 12 \mathrm{~m}$ away (at the sunset side of the

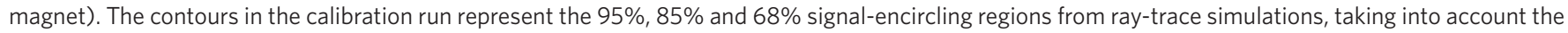

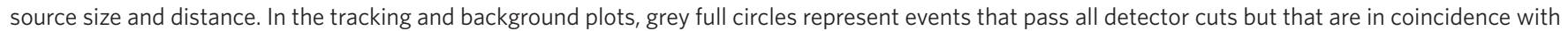

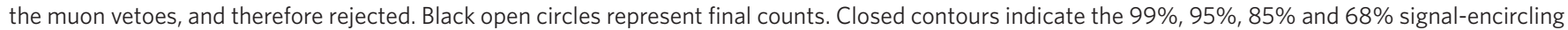

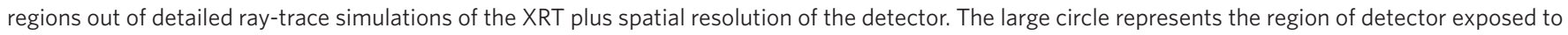
daily energy calibration.

Table 1 | Tracking and background exposure, as well as the integrated 2-7 keV measured count rate, for both tracking and background data, for each of the data sets included in our result.

\begin{tabular}{|c|c|c|c|c|c|c|}
\hline \multirow[t]{2}{*}{ Data set } & \multirow[t]{2}{*}{ Detector } & \multirow[t]{2}{*}{ Year } & \multirow[t]{2}{*}{ Tracking exposure $(h)$} & \multirow[t]{2}{*}{ Background exposure (h) } & \multicolumn{2}{|c|}{$\begin{array}{l}\text { Measured count rates ( } \pm 1 \sigma \text { error) } \\
\left(10^{-6} \mathrm{keV}^{-1} \mathrm{~cm}^{-2} \mathrm{~s}^{-1}\right)\end{array}$} \\
\hline & & & & & Tracking & Background \\
\hline A & SS1 & 2013 & 92.5 & $1,700.0$ & $0.79 \pm 0.18$ & $0.81 \pm 0.04$ \\
\hline B & SS2 & 2013 & 86.5 & $1,407.8$ & $1.37 \pm 0.24$ & $1.48 \pm 0.06$ \\
\hline C & SS1 & 2014 & 118.0 & $1,854.0$ & $0.94 \pm 0.17$ & $1.03 \pm 0.05$ \\
\hline D & SS2 & 2014 & 118.1 & $1,819.6$ & $0.97 \pm 0.18$ & $1.05 \pm 0.05$ \\
\hline E & SS1 & 2015 & 79.5 & $1,237.6$ & $0.77 \pm 0.18$ & $0.89 \pm 0.05$ \\
\hline $\mathrm{F}$ & SS1 & 2015 & 49.7 & 783.1 & $1.77 \pm 0.36$ & $1.65 \pm 0.09$ \\
\hline G & SS1 & 2015 & 83.5 & $1,431.5$ & $1.32 \pm 0.25$ & $1.10 \pm 0.05$ \\
\hline $\mathrm{H}$ & SS2 & 2015 & 81.3 & $1,236.2$ & $0.70 \pm 0.18$ & $0.89 \pm 0.05$ \\
\hline । & $\mathrm{SS} 2$ & 2015 & 51.3 & 800.2 & $1.04 \pm 0.27$ & $1.59 \pm 0.08$ \\
\hline J & SS2 & 2015 & 82.0 & $1,409.2$ & $0.91 \pm 0.20$ & $0.90 \pm 0.05$ \\
\hline K & SR & 2014 & 69.8 & $1,379.4$ & 0 counts & $0.25 \pm 0.05$ counts \\
\hline L & $\mathrm{SR}$ & 2015 & 220.4 & $4,125.4$ & 3 counts & $0.77 \pm 0.15$ counts \\
\hline \multicolumn{3}{|c|}{ Total tracking exposure $(\mathrm{h})$ : } & $1,132.6$ & & & \\
\hline
\end{tabular}

Note that for rows $\mathrm{K}$ and $\mathrm{L}$ background levels are expressed in units of total counts in the ( $95 \%$ signal-enclosing) spot area during the corresponding tracking exposure.

its focal point ${ }^{34}$. This experience is particularly valuable to develop a next-generation scaled-up helioscope.

Our results correspond to $1,132.6 \mathrm{~h} \times$ detector of data taken in axion-sensitive conditions (that is, magnet powered and pointing to the Sun) with the aforementioned detectors in 2013, 2014 and 2015. In 2013, only SS detectors were operative, while in 2014 and 2015 both the SS detectors and the new SR system, installed in CAST in September 2014, took data. The data are divided in sets as shown in Table 1, according to detector and the year of the data-taking campaign. The 2015 SS detectors data are further divided into three sets (E, F, G and H, I, J for SS1 and SS2 detectors respectively) due to an accidental variation in the detector configuration: one of the muon vetoes remained inoperative for about one month, leading to a different background rate during that period. Background levels are defined independently for every data set using data acquired during non-tracking periods. These data typically have $\sim 10$ times more exposure than tracking data, and consequently background levels have $\sim 3$ times smaller statistical error bars. Data shown in the tables and figures always refer to levels after processing. Raw data from the Micromegas detectors undergo an offline filtering process, detailed elsewhere ${ }^{32}$, based on topological information of the event (for example, number of ionization clusters recorded in the chamber, or longitudinal and transversal spread of the signal), to keep only signal-like (that is, X-ray-like) events. The effect of this filtering on raw background levels at low energies is about a factor of 100 , while the signal efficiency stays at $60-70 \%$, depending on the event energy, as determined experimentally by careful calibrations with an X-ray tube at different representative energies ${ }^{38}$. In addition, an anti-coincidence condition with the external plastic muon vetoes is applied, leading to an additional rejection factor of $\sim 2$ (see Fig. 3).

The energy range of interest (RoI) is set between 2 and $7 \mathrm{keV}$, the band that contains most of the expected signal. The low-energy bound is safely above the effective energy threshold of the detectors (which are around $\sim 1$ and $\sim 1.5 \mathrm{keV}$, respectively for SR and SS detectors), and the high-energy bound prevents contamination from the prominent $\sim 8 \mathrm{keV} \mathrm{Cu}$ fluorescence peak observed in the background. The measured tracking and background levels, integrated in this RoI, are presented in Table 1 for each of the data sets. Figure 4 shows the spectral distribution of all SS data sets. The background spectra of the two SR data sets are shown in 
Data set A

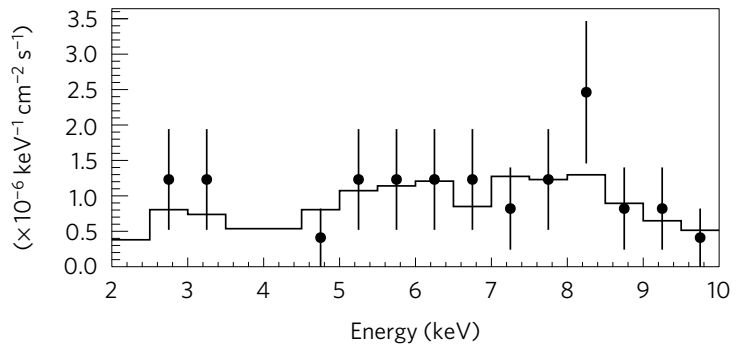

Data set C

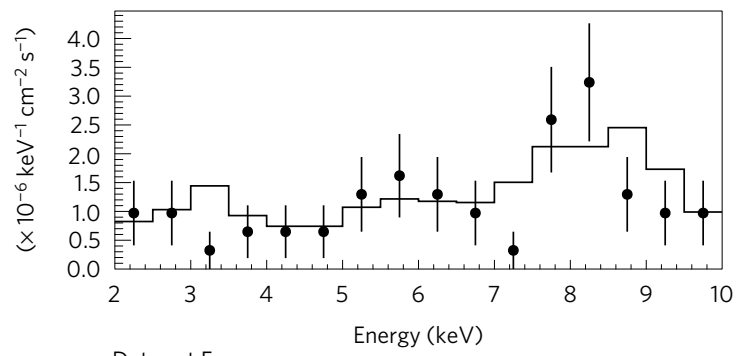

Data set $\mathrm{E}$

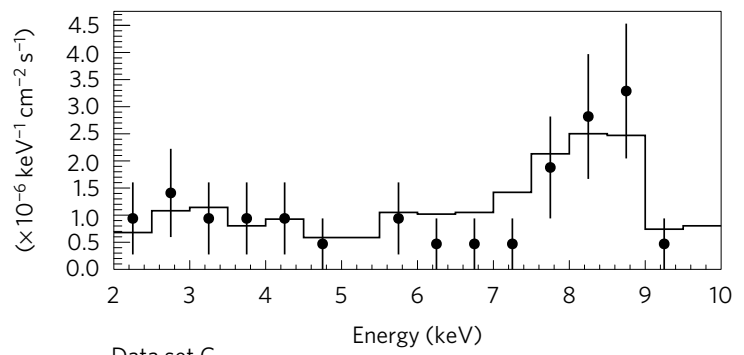

Data set G

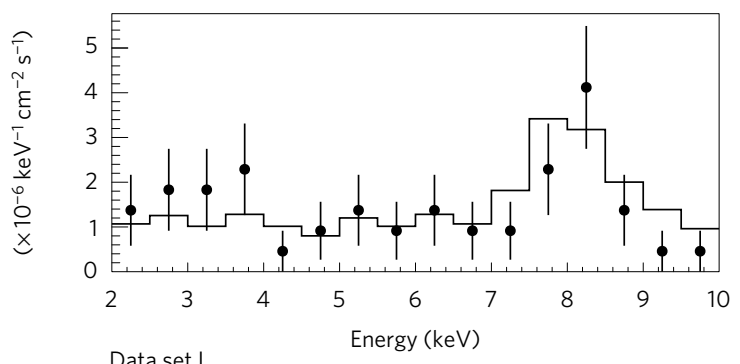

Data set I

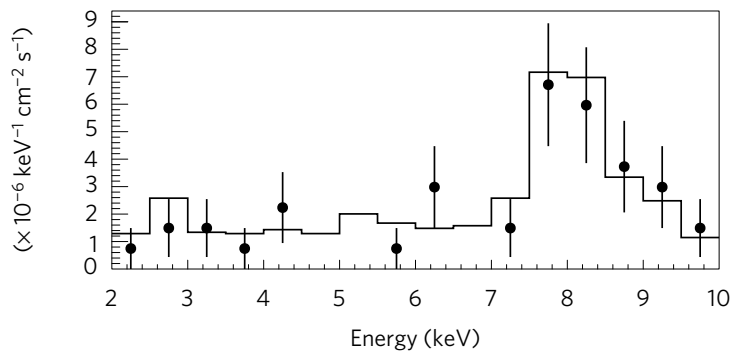

Data set B
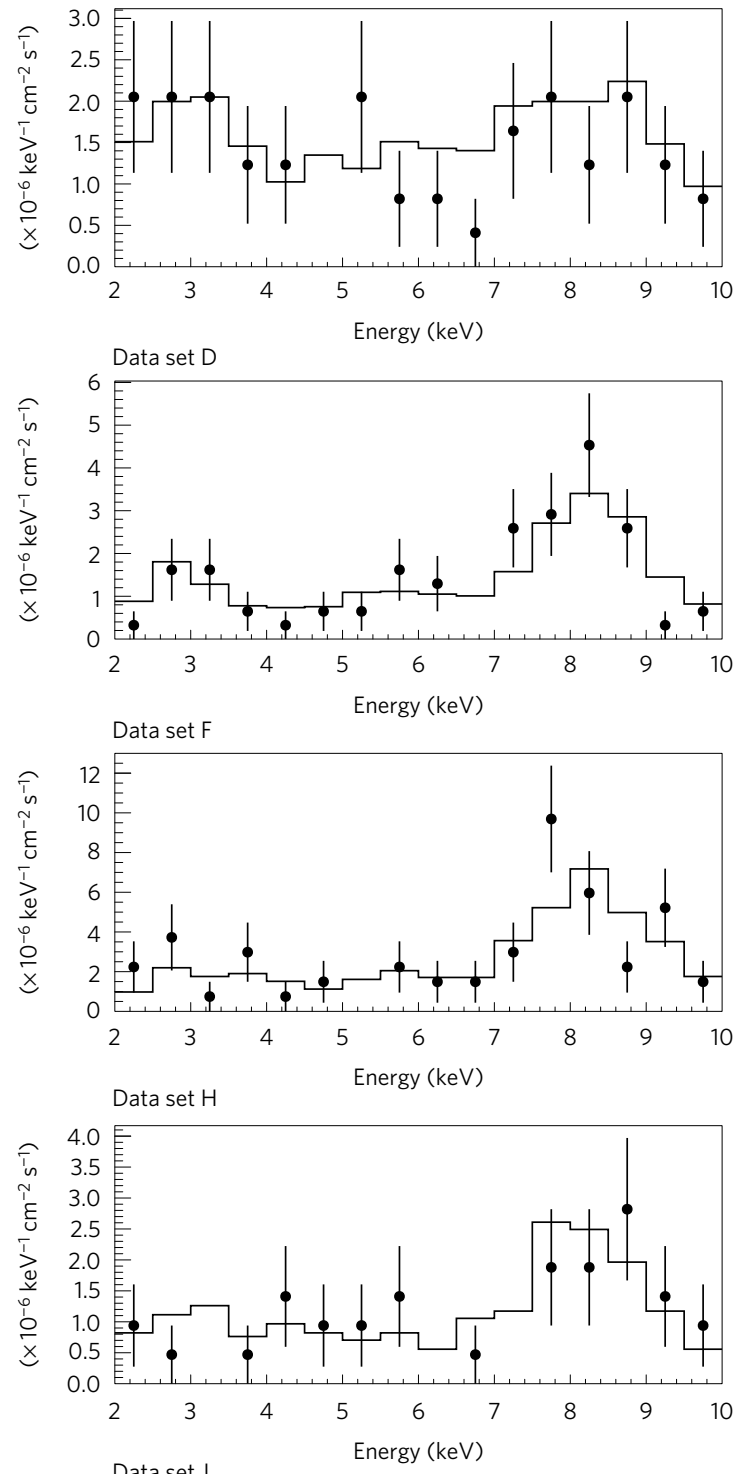

Data set J

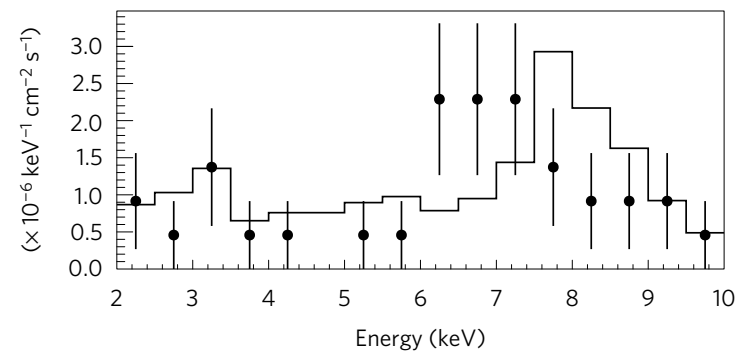

Figure 4 | Measured count rate spectra of tracking (dots) and background (solid line) data for each of the sunset datasets (A-J). The error bars correspond to the 1- $\sigma$ statistical fluctuation of each bin content following Poissonian statistics. The error bars of the background data are omitted for the sake of clarity, although they are typically $\sim 3$ times smaller than the tracking data error bars.

Fig. 5. In these latter plots, data from all the detector area-also outside the signal spot-are included to increase the statistics of the spectra; however, only data from the spot area are used to define the background in the analysis. These measured background levels are primarily attributed to cosmic muon-induced secondaries that are not properly tagged (the muon veto coverage of the shielding is not complete due to spatial constraints of the CAST setups) as well as a remaining environmental $\gamma$-induced background population that reaches the detector despite the shielding, probably associated with the small solid angle facing the magnet that is not possible to shield.
This insight is corroborated by the $\sim 3 \mathrm{keV} \mathrm{Ar}$ and $\sim 8 \mathrm{keV} \mathrm{Cu}$ fluorescence peaks observed in the background spectra (better seen in the SR plot of Fig. 5, due to the better energy resolution of this detector), and will be the basis of future improvements of the detector ${ }^{33}$.

Figure 3 shows the 2D distribution of detected events, both in background and tracking data, in the SR detector, superimposed on the region where the signal is expected. The background level in the signal area could be estimated using the data outside the spot or the data in the spot in non-tracking periods. This second method is preferable, as the background level shows a slight increase at the 

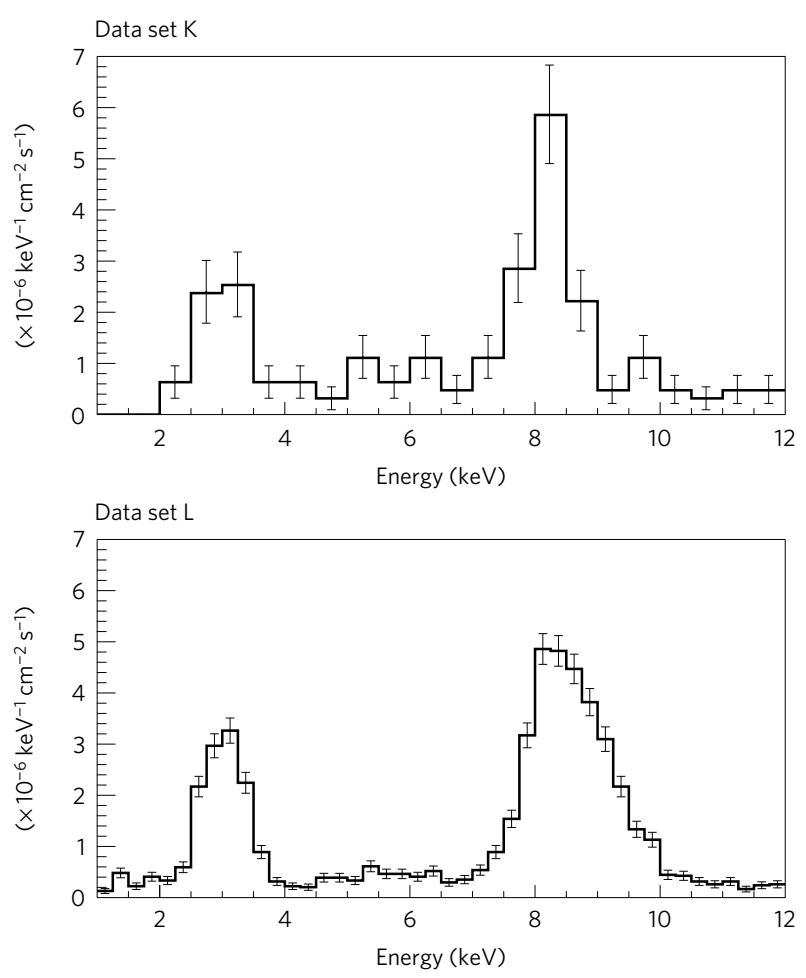

Figure 5 | Measured count rate spectrum of background data in the sunrise detector (both $K$ and $L$ datasets). The error bars correspond to the 1- $\sigma$ statistical fluctuation of each bin content following Poissonian statistics.

centre of the detector (attributed to the detector and shielding geometry). When normalized to the $290 \mathrm{~h}$ of tracking data available (data sets $\mathrm{K}$ and $\mathrm{L}$ in Table 1$)$ only $1.02 \pm 0.22(2.13 \pm 0.47)$ background counts are expected in the 95\% (99\%) signal-enclosing focal spot region, where errors indicate $1-\sigma$ intervals. The tracking data reveal 3 (4) observed counts inside such regions. Their measured energies are $3.05,2.86,2.94$ and $2.56 \mathrm{keV}$.

\section{Data analysis and results}

The data analysis follows similar previous analyses of CAST data $^{14-16}$. We define an unbinned likelihood function

$$
\log \mathcal{L} \propto-R_{T}+\sum_{i}^{n} \log R\left(E_{i}, d_{i}, \mathbf{x}_{i}\right)
$$

where $R_{T}$ is the expected number of counts from the axion-tophoton conversion in all data sets, integrated over the tracking exposure time and energy of interest. The sum is over each of the $n$ detected counts in the energy RoI during the tracking time, for an expected rate $R\left(E_{i}, d_{i}, \mathbf{x}_{i}\right)$ as a function of the energy $E_{i}$, data set $d_{i}$, and detector coordinates $\mathbf{x}_{i}$ of the event $i$, and given by the expression

$$
R(E, d, \mathbf{x})=B(E, d)+S(E, d, \mathbf{x})
$$

where $B(E, d)$ is the background level for data set $d$, considered constant in time and $\mathbf{x}_{i}$ within the data set. $S(E, d, \mathbf{x})$ is the expected rate from axion conversion in the detector of data set $d$ given by

$$
S(E, d, \mathbf{x})=\frac{\mathrm{d} \Phi_{a}}{\mathrm{~d} E} P_{a \rightarrow \gamma} \epsilon(d, E, \mathbf{x})
$$

Here, $\epsilon(d, E, \mathbf{x})$ is the detector response for data set $d$, and includes both the $E$-dependent detector efficiency (both hardware and software), and for the SR system also the $E$-dependent optics throughput and the expected signal distribution over $\mathbf{x}$ due to the optics PSF shown in Fig. 3. For the SS detector there is no such dependency and $\epsilon(d, E, \mathbf{x})=\epsilon(d, E)$.

Finally, $\mathrm{d} \Phi_{a} / d E$ is the differential solar axion flux, which can be parameterized by the expression ${ }^{12}$

$$
\frac{\mathrm{d} \Phi_{a}}{\mathrm{~d} E}=6.02 \times 10^{10} g_{10}^{2} \frac{E^{2.481}}{\mathrm{e}^{E / 1.205}}\left[\mathrm{~cm}^{-2} \mathrm{~s}^{-1} \mathrm{keV}^{-1}\right]
$$

with $g_{10}=g_{a \gamma} /\left(10^{-10} \mathrm{GeV}^{-1}\right)$ and energy $E$ in $\mathrm{keV}$. The axion-tophoton conversion probability $P_{a \rightarrow \gamma}$ was given in equation (1).

By numerically maximizing $\log \mathcal{L}$ a best-fit value $g_{10, \min }^{4}$ is obtained. This value is compatible with the absence of a signal in the entire axion mass range, and thus an upper limit on $g_{a \gamma}$ is extracted, by integrating the Bayesian posterior probability density function (PDF) from zero up to $95 \%$ of the total PDF area, using a flat prior in $g_{10}^{4}$ for positive values, and zero for the unphysical negative ones. The computed upper limit for several values of $m_{a}$ is displayed in red in Fig. 2.

For mass values below $m_{a} \lesssim 0.02 \mathrm{eV}$, the mass independent bestfit value is $g_{10 \text { min }}^{4}=\left(-0.06_{-0.07}^{+0.10}\right)$, where the errors indicate $1-\sigma$ intervals. The upper limit in this mass range constitutes our main result:

$$
g_{a \gamma}<0.66 \times 10^{-10} \mathrm{GeV}^{-1} \text { at } 95 \% \mathrm{CL}
$$

This constraint considers only statistical fluctuations in the tracking data. Other potentially important systematic effects would include uncertainties in input parameters such as the magnet length and strength, the background levels, the tracking accuracy, the optical alignment of the SR system, and the theoretical uncertainty of the expected signal. All these contributions have been estimated to be negligible in the final result, which is dominated by the statistical error of the low-counting observation. Overall systematic effects are well below $10 \%$ of the quoted result. It is worth noting that the observation of the SR counts represents a statistical fluctuation that slightly worsens the final result with respect to the expected sensitivity (defined as the average exclusion of an ensemble of possible statistical outcomes under the background-only hypothesis), estimated as $g_{10 \text {,average }} \lesssim 0.64$.

\section{Discussion}

The final solar axion run of CAST has provided the new constraint of equation (6) on the axion-photon coupling strength for $m_{a} \lesssim 0.02 \mathrm{eV}$. It is shown in the wider $m_{a}-g_{a \gamma}$ landscape in Fig. 6. In particular, axions and photons can also interconvert in astrophysical $B$ fields that tend to be much weaker than in CAST, but extend over much larger distances. For example, the non-observation of $\gamma$ rays contemporaneous with the SN 1987A neutrino signal provides a limit $g_{10} \lesssim 0.053$ for $m_{a} \lesssim 0.44 \mathrm{neV}$ (ref. 39). This conversion effect could be important also for other astrophysical and cosmological sources, especially for the propagation of $\mathrm{TeV} \gamma$ rays and as an explanation for the soft X-ray excess in galaxy clusters (for a recent review see ref. 40). In the very low mass domain, propagation experiments for laser beams in magnetic fields have also explored the $g_{a \gamma}-m_{a}$ parameter space ${ }^{41,42}$.

Helioscopes using the Bragg technique can reach larger axion masses than CAST because the electric fields in the crystals are inhomogeneous on much smaller scales. Yet because of their limited size, even the best case shown in Fig. 6 from DAMA ${ }^{19}$ is not competitive. In the future, CUORE may reach values of $g_{a \gamma}$ comparable to the CAST ${ }^{3} \mathrm{He}$ limits near $m_{a} \sim 1 \mathrm{eV}$, but extended to larger masses ${ }^{43}$.

However, QCD axions in this parameter range would thermalize in the early universe and provide a hot dark-matter fraction. Cosmology provides a constraint corresponding to $m_{a}<0.86 \mathrm{eV}$ (vertical dashed red line in Fig. 6), which in the future may reach $m_{a}<0.15 \mathrm{eV}$ (ref. 44). Therefore, exploring beyond the $1 \mathrm{eV}$ mass range is relevant only for those axion-like particles which, unlike QCD axions, do not thermalize efficiently. 


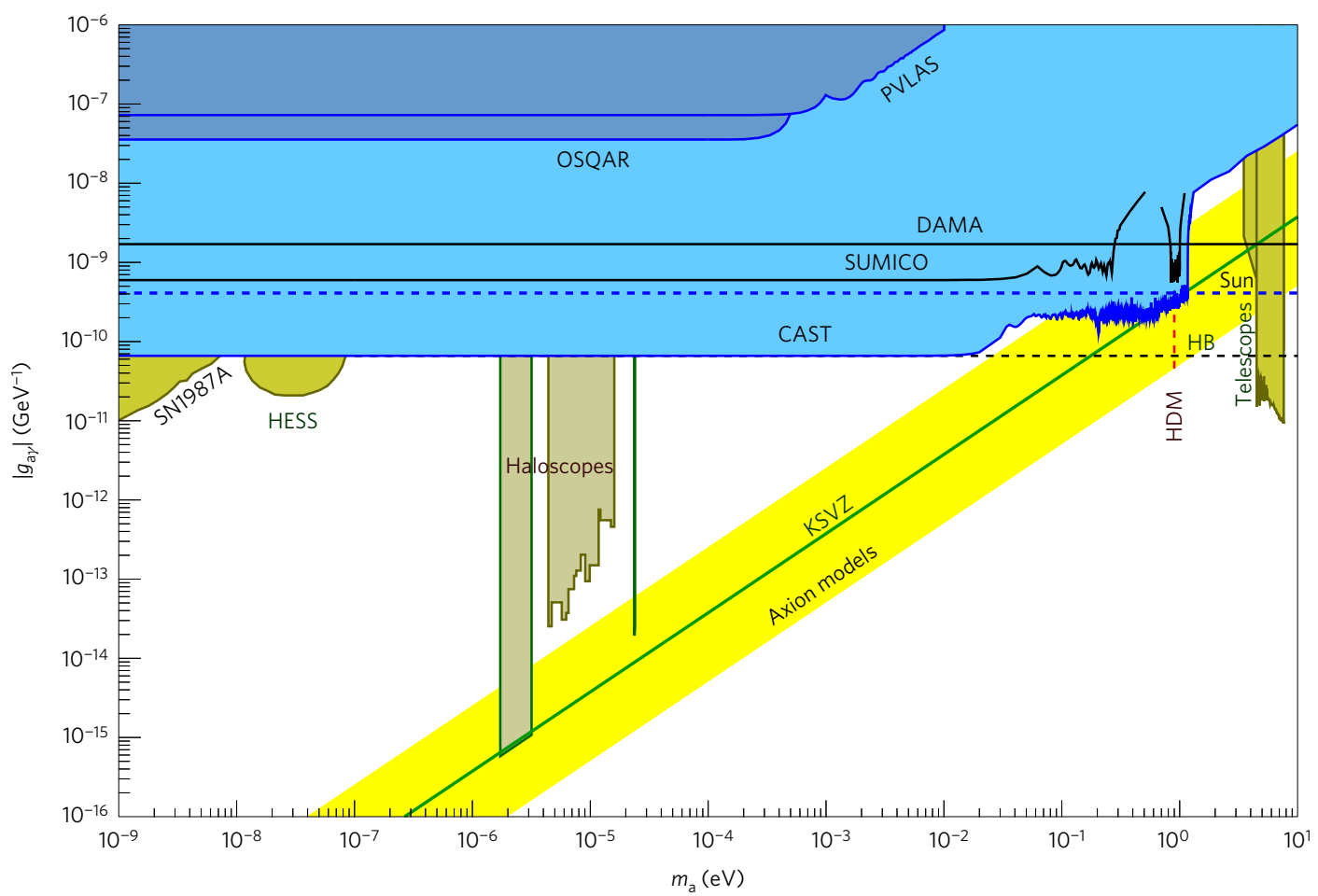

Figure 6 | Constraints on the two-photon coupling $g_{a y}$ of axions and similar particles depending on their mass $m_{a}$. Apart from the CAST limits updated with the result presented here, we show the results from the previous helioscope Sumico and the best limit from the Bragg technique (DAMA). Moreover, we show the latest limits from the laser propagation experiments OSQAR and PVLAS, high-energy photon propagation in astrophysical B-fields (H.E.S.S.), the SN1987A observation, and telescope limits for cosmic axion decay lines. Horizontal dashed lines provide limits from properties of the Sun and the energy loss of horizontal branch (HB) stars. The vertical dashed line denotes the cosmic hot dark-matter (HDM) limit which only applies to QCD axions. The haloscope limits assume that axions are the galactic dark matter. The yellow band of QCD axion models and the green KSVZ line are as in Fig. 2.

Solar axion searches usually assume that the axion flux is only a small perturbation of the Sun. Actually for $g_{10} \gtrsim 20$ axion losses are so large that one cannot construct self-consistent solar models $s^{45}$. Moreover, the measured solar neutrino flux and helioseismology require $g_{10}<4.1$ at $3 \sigma$ confidence ${ }^{46}$ (dashed blue line in Fig. 6), implying that CAST is the only solar axion search that has gone beyond this recent limit.

A sensitivity comparable to the new CAST limit derives from traditional stellar energy-loss arguments. In particular, Primakoff losses accelerate the helium-burning phase of horizontal branch (HB) stars, reducing their number count relative to low-mass red giants, $R=N_{\mathrm{HB}} / N_{\mathrm{RGB}}$, in globular clusters (see dashed line labelled 'HB' in Fig. 6). The most recent analysis finds $g_{10}<0.66$ (95\% CL) and actually a mild preference for $g_{10} \sim 0.4$, although a detailed budget of systematic uncertainties is not currently available ${ }^{7}$.

Solar axion searches beyond CAST, and at the same time beyond the HB star limit as a benchmark, require a new effort on a much larger scale, for example the proposed helioscope IAXO ${ }^{28}$. For small masses, new regions in the $g_{a \gamma}-m_{a}$ will be explored with the upcoming ALPS-II laser propagation experiment at $\mathrm{DESY}^{47}$, by higher-statistics $\mathrm{TeV} \gamma$-ray observations, or the $\gamma$-ray signal from a future galactic supernova ${ }^{40}$. Beyond the $g_{a \gamma}-m_{a}$ parameter space, many attractive detection opportunities are pursued worldwide, notably under the assumption that axions are the cosmic dark matter ${ }^{4}$.

After finishing its solar axion programme in 2015, CAST itself has turned to a broad physics programme at the low-energy frontier. In particular, this includes KWISP, a sensitive force detector, and an InGrid detector, both in search of solar chameleons, as well as CASTCAPP and RADES, implementing long-aspect-ratio microwave cavities in the CAST magnet to search for dark-matter axions around $m_{a} \sim 20 \mu \mathrm{eV}$ (ref. 48 ).
Whichever of the many new developments worldwide will lead to the detection of axions or similar particles in the $g_{a \gamma}-m_{a}$ parameter space, our new CAST result will remain the benchmark for many years to come and guide future explorations.

Data availability. The data that support the plots within this paper and other findings of this study are available from the corresponding author upon reasonable request.

Received 25 January 2017; accepted 23 March 2017; published online 1 May 2017

\section{References}

1. Patrignani, C. et al. (Particle Data Group) Review of particle physics. Chin. Phys. C 40, 100001 (2016).

2. Jaeckel, J. \& Ringwald, A. The low-energy frontier of particle physics. Annu. Rev. Nucl. Part. Sci. 60, 405-437 (2010).

3. Kuster, M., Raffelt, G. \& Beltrán, B. (eds) Axions: Theory, Cosmology, and Experimental Searches Lecture Notes in Physics Vol. 741, 1-237 (2008).

4. Graham, P. W., Irastorza, I. G., Lamoreaux, S. K., Lindner, A. \& van Bibber, K. A. Experimental searches for the axion and axion-like particles. Annu. Rev. Nucl. Part. Sci. 65, 485 (2015).

5. Arvanitaki, A. \& Geraci, A. A. Resonantly detecting axion-mediated forces with nuclear magnetic resonance. Phys. Rev. Lett. 113, 161801 (2014).

6. Brito, R., Cardoso, V. \& Pani, P. Superradiance: energy extraction, black-hole bombs and implications for astrophysics and particle physics. Lect. Notes Phys. 906, 1-237 (2015).

7. Ayala, A., Domínguez, I., Giannotti, M., Mirizzi, A. \& Straniero, O. Revisiting the bound on axion-photon coupling from globular clusters. Phys. Rev. Lett. 113, 191302 (2014).

8. Giannotti, M., Irastorza, I., Redondo, J. \& Ringwald, A. Cool WISPs for stellar cooling excesses. J. Cosmol. Astropart. Phys. 1605, 057 (2016).

9. Sikivie, P. Experimental tests of the invisible axion. Phys. Rev. Lett. 51, 1415-1417 (1983); erratum 52, 695 (1984). 
10. Raffelt, G. \& Stodolsky, L. Mixing of the photon with low mass particles. Phys. Rev. D 37, 1237-1249 (1988).

11. Zioutas, K. et al. (CAST Collaboration) First results from the CERN Axion Solar Telescope (CAST). Phys. Rev. Lett. 94, 121301 (2005).

12. Andriamonje, S. et al. (CAST Collaboration) An improved limit on the axion-photon coupling from the CAST experiment. J. Cosmol. Astropart. Phys. 0704, 010 (2007).

13. Arik, E. et al. (CAST Collaboration) Probing eV-scale axions with CAST. J. Cosmol. Astropart. Phys. 0902, 008 (2009).

14. Arik, M. et al. (CAST Collaboration) New solar axion search using the CERN Axion Solar Telescope with ${ }^{4} \mathrm{He}$ filling. Phys. Rev. D 92, 021101 (2015).

15. Arik, M. et al. (CAST Collaboration) CAST search for sub-eV mass solar axions with ${ }^{3}$ He buffer gas. Phys. Rev. Lett. 107, 261302 (2011).

16. Arik, M. et al. (CAST Collaboration) CAST solar axion search with ${ }^{3} \mathrm{He}$ buffer gas: closing the hot dark matter gap. Phys. Rev. Lett. 112, 091302 (2014).

17. Inoue, Y. et al. Search for solar axions with mass around $1 \mathrm{eV}$ using coherent conversion of axions into photons. Phys. Lett. B 668, 93-97 (2008).

18. Paschos, E. A. \& Zioutas, K. A proposal for solar axion detection via Bragg scattering. Phys. Lett. B 323, 367-372 (1994).

19. Bernabei, R. et al. Search for solar axions by Primakoff effect in NaI crystals. Phys. Lett. B 515, 6-12 (2001).

20. Andriamonje, S. et al. (CAST Collaboration) Search for $14.4-\mathrm{keV}$ solar axions emitted in the M1-transition of Fe-57 nuclei with CAST. J. Cosmol. Astropart. Phys. 0912, 002 (2009).

21. Andriamonje, S. et al. (CAST Collaboration) Search for solar axion emission from ${ }^{7} \mathrm{Li}$ and $\mathrm{D}(p, \gamma)^{3} \mathrm{He}$ nuclear decays with the CAST $\gamma$-ray calorimeter. J. Cosmol. Astropart. Phys. 1003, 032 (2010).

22. Barth, K. et al. (CAST Collaboration) CAST constraints on the axion-electron coupling. J. Cosmol. Astropart. Phys. 1305, 010 (2013).

23. Anastassopoulos, V. et al. (CAST Collaboration) Search for chameleons with CAST. Phys. Lett. B 749, 172-180 (2015).

24. Redondo, J. Atlas of solar hidden photon emission. J. Cosmol. Astropart. Phys 1507, 024 (2015)

25. Autiero, D. et al. The CAST time projection chamber. New J. Phys. 9, 171 (2007).

26. Abbon, P. et al. The Micromegas detector of the CAST experiment. New J. Phys. 9, 170 (2007)

27. Kuster, M. et al. The X-ray Telescope of CAST. New J. Phys. 9, 169 (2007)

28. Armengaud, E. et al. Conceptual design of the International Axion Observatory (IAXO). JINST 9, T05002 (2014).

29. Zioutas, K. et al. A Decommissioned LHC model magnet as an axion telescope. Nucl. Instrum. Meth. A 425, 480-489 (1999).

30. Giomataris, Y., Rebourgeard, P., Robert, J. P. \& Charpak, G. MICROMEGAS: a high granularity position sensitive gaseous detector for high particle flux environments. Nucl. Instrum. Meth. A 376, 29-35 (1996).

31. Andriamonje, S. et al. Development and performance of Microbulk Micromegas detectors. JINST 5, P02001 (2010).

32. Aune, S. et al. Low background X-ray detection with Micromegas for axion research. JINST 9, P01001 (2014).

33. Irastorza, I. G. et al. Gaseous time projection chambers for rare event detection results from the T-REX project. II. Dark matter. J. Cosmol. Astropart. Phys. 1601, 034 (2016); erratum 1605, E01 (2016).

34. Aznar, F. et al. A Micromegas-based low-background X-ray detector coupled to a slumped-glass telescope for axion research. J. Cosmol. Astropart. Phys. 1512, 008 (2015).

35. Koglin, J. E. et al. NuSTAR hard X-ray optics design and performance. Proc. SPIE Int. Soc. Opt. Eng. 7437, 74370C (2009).

36. Harrison, F. A. et al. The nuclear spectroscopic telescope array (NuSTAR) high-energy X-ray mission. Astrophys. J. 770, 103 (2013).

37. Jakobsen, A. C., Pivovaroff, M. J. \& Christensen, F. E. X-ray optics for axion helioscopes. Proc. SPIE Int. Soc. Opt. Eng. 8861, 886113 (2013)

38. Gracia Garza, J. Micromegas for the Search of Solar Axions in CAST and Low-Mass WIMPs in TREX-DM (CERN, 2015); https://cds.cern.ch/record/2125276? ln=es

39. Payez, A. et al. Revisiting the SN1987A gamma-ray limit on ultralight axion-like particles. J. Cosmol. Astropart. Phys. 1502, 006 (2015).

40. Meyer, M., Giannotti, M., Mirizzi, A., Conrad, J. \& Sanchez-Conde, M. A. Fermi Large Area Telescope as a galactic supernovae axionscope. Phys. Rev. Lett. 118, 011103 (2017).

41. Ballou, R. et al. (OSQAR Collaboration) New exclusion limits on scalar and pseudoscalar axionlike particles from light shining through a wall. Phys. Rev. D 92, 092002 (2015).
42. Della, F. et al. The PVLAS experiment: measuring vacuum magnetic birefringence and dichroism with a birefringent Fabry Perot cavity. Eur. Phys. J. C 76, 24 (2016).

43. Li, D., Creswick, R. J., Avignone, F. T. \& Wang, Y. Theoretical estimate of the sensitivity of the CUORE detector to solar axions. J. Cosmol. Astropart. Phys. 1510, 065 (2015).

44. Archidiacono, M., Hannestad, S., Mirizzi, A., Raffelt, G. \& Wong, Y. Y. Y. Axion hot dark matter bounds after Planck. J. Cosmol. Astropart. Phys. 1310, 020 (2013).

45. Schlattl, H., Weiss, A. \& Raffelt, G. Helioseismological constraint on solar axion emission. Astropart. Phys. 10, 353-359 (1999).

46. Vinyoles, N. et al. New axion and hidden photon constraints from a solar data global fit. J. Cosmol. Astropart. Phys. 1510, 015 (2015)

47. Bähre, R. et al. Any light particle search II-Technical design report. JINST 8, T09001 (2013)

48. Desch, K. (CAST collaboration) CAST Status Report to the SPSC for the 123rd Meeting (CERN, 2016); http://cds.cern.ch/record/2221945?ln=en

49. Di Luzio, L., Mescia, F. \& Nardi, E. Redefining the axion window. Phys. Rev. Lett. 118, 031801 (2017).

\section{Acknowledgements}

We thank CERN for hosting the experiment and for technical support to operate the magnet and cryogenics. We thank V. Burwitz, G. Hartner of the MPE PANTER X-ray test facility for providing the opportunity to calibrate the X-ray telescope and for assistance in collecting and analysing the characterization data. We acknowledge support from NSERC (Canada), MSE (Croatia) and Croatian Science Foundation under the project IP-2014-09-3720, CEA (France), BMBF (Germany) under the grant numbers 05 CC2EEA/9 and 05 CC1RD1/0, DFG (Germany) under grant numbers HO 1400/7-1 and EXC-153, GSRT (Greece), NSRF: Heracleitus II, RFFR (Russia), the Spanish Ministry of Economy and Competitiveness (MINECO) under Grants No. FPA2011-24058 and No. FPA2013-41085-P (grants partially funded by the European Regional Development Fund, ERDF/FEDER), the European Research Council (ERC) under grant ERC-2009-StG-240054 (T-REX), Turkish Atomic Energy Authority (TAEK), NSF (USA) under Award No. 0239812, NASA under the grant number NAG5-10842, and IBS (Korea) with code IBS-R017-D1-2017-a00. Part of this work was performed under the auspices of the US Department of Energy by Lawrence Livermore National Laboratory under Contract No. DE-AC52-07NA27344.

\section{Author contributions}

S.A., J.F.C., T.D., I.G., E.F.-R., F.J.I., J.A.G., I.G.I., J.G.G and T.P. conceived, designed and built the Micromegas X-ray detectors. F.C., T.A.D., C.J.H., A.J., M.J.P., J.R. and J.K.V. conceived, designed, calibrated and built the $\mathrm{X}$-ray telescope. J.F.C., T.D., F.J.I., J.A.G., J.G.G., E.R.C. and J.R. installed, calibrated and operated optics and detectors in the experiment. T.D, F.J.I., J.A.G., J.G.G., I.G.I. and G.L. conceived and implemented the low-background measures on the detection systems. K.B., M.D., J.M.L., L.S., T.V., S.C.Y. and K.Z. operated and maintained the magnet, vacuum, slow control and other ancillary systems, as well as the interfaces with the detection systems. M.D., T.V. and M.K. organized the periodical pointing calibration of the tracking system. B.L., T.V., M.D. and K.Z. organized the data-taking runs and the general operation of the experiment. M.J.P. performed the optics ray-trace models. J.A.G., F.J.I. and I.G.I. monitored and analysed the detector data. I.G.I. coordinated the high level analysis and made the plots. I.G.I., B.L., M.J.P. and G.R. wrote the manuscript. All authors contributed to the data-taking shifts and the operation and maintenance of the experiment at a whole, and all authors commented on the manuscript.

\section{Additional information}

Reprints and permissions information is available online at www.nature.com/reprints. Publisher's note: Springer Nature remains neutral with regard to jurisdictional claims in published maps and institutional affiliations. Correspondence and requests for materials should be addressed to I.G.I.

\section{Competing financial interests}

The authors declare no competing financial interests.

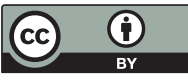

This article is licensed under a Creative Commons Attribution 4.0 International License, which permits use, sharing, adaptation, distribution and reproduction in any medium or format, as long as you give appropriate credit to the original author(s) and the source, provide a link to the Creative Commons license, and indicate if changes were made.

The images or other third party material in this article are included in the article's Creative Commons license, unless indicated otherwise in a credit line to the material. If material is not included in the article's Creative Commons license and your intended use is not permitted by statutory regulation or exceeds the permitted use, you will need to obtain permission directly from the copyright holder. To view a copy of this license, visit http://creativecommons.org/licenses/by/4.0/. 


\section{CAST Collaboration}

V. Anastassopoulos', S. Aune ${ }^{2}$, K. Barth ${ }^{3}$, A. Belov ${ }^{4}$, H. Bräuninger ${ }^{5}$, G. Cantatore ${ }^{6}$, J. M. Carmona ${ }^{7}$, J. F. Castel ${ }^{7}$, S. A. Cetin ${ }^{8}$, F. Christensen ${ }^{9}$, J. I. Collarr ${ }^{10}$, T. Dafni ${ }^{7}$, M. Davenport ${ }^{3}$, T. A. Decker ${ }^{11}$, A. Dermenev ${ }^{4}$, K. Desch $^{12}$, C. Eleftheriadis ${ }^{13}$, G. Fanourakis ${ }^{14}$, E. Ferrer-Ribas' ${ }^{2}$, H. Fischer ${ }^{15}$, J. A. García ${ }^{7 \dagger}$, A. Gardikiotis ${ }^{1}$, J. G. Garza ${ }^{7}$, E. N. Gazis ${ }^{16}$, T. Geralis ${ }^{14}$,

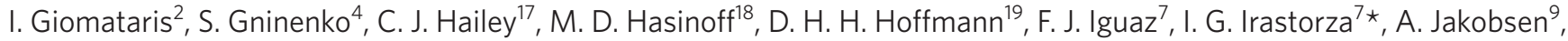
J. Jacoby ${ }^{20}$, K. Jakovčić ${ }^{21}$, J. Kaminski ${ }^{12}$, M. Karuza ${ }^{6,22 \dagger}$, N. Kralj2 ${ }^{2 \dagger}$, M. Krčmar ${ }^{21}$, S. Kostoglou ${ }^{3}$, Ch. Krieger ${ }^{12}$, B. Lakić ${ }^{21}$,

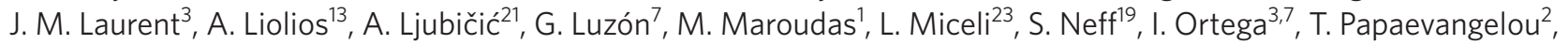
K. Paraschou ${ }^{13}$, M. J. Pivovaroff'11, G. Raffelt ${ }^{24}$, M. Rosu ${ }^{19 \dagger}$, J. Ruz ${ }^{11}$, E. Ruiz Chóliz ${ }^{7}$, I. Savvidis ${ }^{13}$, S. Schmidt ${ }^{12}$, Y. K. Semertzidis ${ }^{23 \dagger}$, S. K. Solanki ${ }^{25 \dagger}$, L. Stewart ${ }^{3}$, T. Vafeiadis ${ }^{3}$, J. K. Vogel ${ }^{11}$, S. C. Yildiz ${ }^{8 \dagger}$, K. Zioutas K $^{1,3}$

\footnotetext{
${ }^{1}$ Physics Department, University of Patras, 26500 Patras, Greece. ${ }^{2}$ IRFU, CEA, Université Paris-Saclay, 91191 Gif-sur-Yvette, France. ${ }^{3}$ European Organization for Nuclear Research (CERN), CH-1211 Genève, Switzerland. ${ }^{4}$ Institute for Nuclear Research (INR), Russian Academy of Sciences, 117312 Moscow, Russia. ${ }^{5}$ Max-Planck-Institut für Extraterrestrische Physik, D-85741 Garching, Germany. ${ }^{6}$ Istituto Nazionale di Fisica Nucleare (INFN), Sezione di Trieste and Università di Trieste, 34127 Trieste, Italy. ${ }^{7}$ Grupo de Investigación de Física Nuclear y Astropartículas, Universidad de Zaragoza, 50009 Zaragoza, Spain. ${ }^{8}$ Istanbul Bilgi University, High Energy Physics Research Center, Eyup, Istanbul 34060, Turkey. ${ }^{9}$ DTU Space, National Space Institute, Technical University of Denmark, 2800 Lyngby, Denmark. ${ }^{10}$ Enrico Fermi Institute and KICP, University of Chicago, Chicago, Illinois 60637, USA. ${ }^{11}$ Lawrence Livermore National Laboratory, Livermore, California 94550, USA. ${ }^{12}$ Physikalisches Institut, University of Bonn, 53115 Bonn, Germany. ${ }^{13}$ Aristotle University of Thessaloniki, 54124 Thessaloniki, Greece. ${ }^{14}$ National Center for Scientific Research "Demokritos", 15310 Athens, Greece. ${ }^{15}$ Albert-Ludwigs-Universität Freiburg, 79085 Freiburg, Germany. ${ }^{16}$ National Technical University of Athens, 15780 Athens, Greece. ${ }^{17}$ Physics Department and Columbia Astrophysics Laboratory, Columbia University, New York, New York 10027, USA. ${ }^{18}$ Department of Physics and Astronomy, University of British Columbia, Vancouver V6T 1Z1, Canada. ${ }^{19}$ Technische Universität Darmstadt, IKP, 64289 Darmstadt, Germany. ${ }^{20}$ Johann Wolfgang Goethe-Universität, Institut für Angewandte Physik, 60438 Frankfurt am Main, Germany. ${ }^{21}$ Rudjer Bošković Institute, 10000 Zagreb, Croatia. ${ }^{22}$ Department of Physics and Centre for Micro and Nano Sciences and Technologies, University of Rijeka, 51000 Rijeka, Croatia. ${ }^{23}$ Center for Axion and Precision Physics Research, Institute for Basic Science (IBS), Daejeon 34141, Republic of Korea. ${ }^{24}$ Max-Planck-Institut für Physik (Werner-Heisenberg-Institut), 80805 München, Germany. ${ }^{25}$ Max-Planck-Institut für Sonnensystemforschung, 37077 Göttingen, Germany. †Present addresses: Institute of High Energy Physics, Chinese Academy of Sciences, 100049 Beijing (J.A.G.); Photonics and Quantum Optics Unit, Center of Excellence for Advanced Materials and Sensing Devices, University of Rijeka, 51000 Rijeka, Croatia (M.K.); Physics Division, School of Sciences and Technology, University of Camerino, I-62032 Camerino, Italy (N.K.); Extreme Light Infrastructure - Nuclear Physics (ELI-NP), 077125 Magurele, Romania (M.R.); Department of Physics, Korea Advanced Institute of Science and Technology (KAIST), Daejeon 34141, Republic of Korea (Y.K.S.); School of Space Research, Kyung Hee University, Yongin 446-701, Republic of Korea (S.K.S.); Department of Physics and Astronomy, University of California, Irvine, California 92697, USA (S.C.Y.). *e-mail: igor.irastorza@cern.ch
} 